\title{
Effect of decision feedback equalizer taps on 3x6- channel mode-wavelength division multiplexing system performance in multimode fiber
}

\author{
Tendai Masunda ${ }^{1, *}$, Angela Amphawan ${ }^{1,2}$, and Aras Al-dawoodi ${ }^{3}$ \\ ${ }^{1}$ School of Computing, Universiti Utara Malaysia Sintok, Kedah, Malaysia \\ ${ }^{2}$ Research Laboratory of Electronics, Massachusetts Institute of Technology,Cambridge, Massachusetts, USA. \\ ${ }^{3}$ Computer Science Department, College of Science, Kirkuk University, 00964 Kirkuk, Iraq
}

\begin{abstract}
In this paper, the effect of tap configurations in a decision feedback equalizer is investigated for controlling modal dispersion in a $60 \mathrm{~Gb} / \mathrm{s} 18$-channel mode division multiplexing - wavelength division multiplexing system in a $2.5 \mathrm{~km}$ multimode fiber on three Laguerre-Gaussian modes and six wavelengths centered at $1550 \mathrm{~nm}$. Spectrum analyser diagrams and bit error rate measurements show satisfactory performance for feed forward - feedback tap combinations $(10,5),(14,0),(15,6)$ and $(15,10)$ by effectively suppressing higher order modes.
\end{abstract}

\section{Introduction}

Today's optical networks are the backbone of modern communications networks, moving data traffic across distances of just a few kilometers to stretch across continents. There has been an exponential growth in Internet users and the demand for higher bandwidth as users interact more spontaneously on the Internet using applications that require large data transfers and high interactivity such as for video conferencing, video streaming, voice-over-Internet-Protocol (VoIP), television streaming, social media, online gaming and others [1-3]. Digital content is growing and network subscribers require high data transfer and interactivity.

Various multiplexing schemes have been investigated for increasing the bandwidth of current optical networks, such as wavelength division multiplexing (WDM) [4], orthogonal frequency division multiplexing (OFDM) [5], optical code division multiple-access system (OCDMA) [6] and polarization division multiplexing (PDM) [7]. There has also been a compelling vision to accommodate traffic growth by devising ways that can allow us to transmit large amounts of data in a single fiber using modes of an optical fiber. The future solution lies in mode division multiplexing (MDM) which allows independent data streams to be transmitted on multiple modes of an optical fiber, which are then retrieved by multiple receivers [8].

\footnotetext{
*Corresponding author: tendaimasunda@gmail.com
}

\section{Related Work}

MDM complements WDM, OFDM and other multiplexing schemes which have been the workhorse of major telecommunication companies in order to increase the aggregate capacity. MDM has been realized through through holographic wavefront generation [9-12], multi-core fibers [13-15], mode-selective fiber couplers [16-18], laser cavity design [19-21] or via optical signal processing $[17,22,23]$.

The frontier of MDM lies in its prowess to unleash the physical capacity boundaries of multimode fiber (MMF) by confining modal coupling which instigates modal dispersion and inter-symbol interference, thus restricting the achievable bandwidth [24]. To suppress mode coupling, several adaptive equalization schemes have been introduced in MDM. Adaptive equalizers work efficiently in an unknown environment. These filters extract useful information from noisy data and employ recursive algorithms for continuous adjustment within a changing environment for example when signal statistics are not defined priori, and also when there is a spectral overlap between the signal of interest and noisy signal. Equalizers that have been used thus far in MDM include least-mean-square algorithm which reduce the mean square error between the received and target channel impulse response [25], recursive least-squares which recursively finds the coefficients that minimize a weighted linear least squares cost function relating to the received and target channel impulse response [26], 


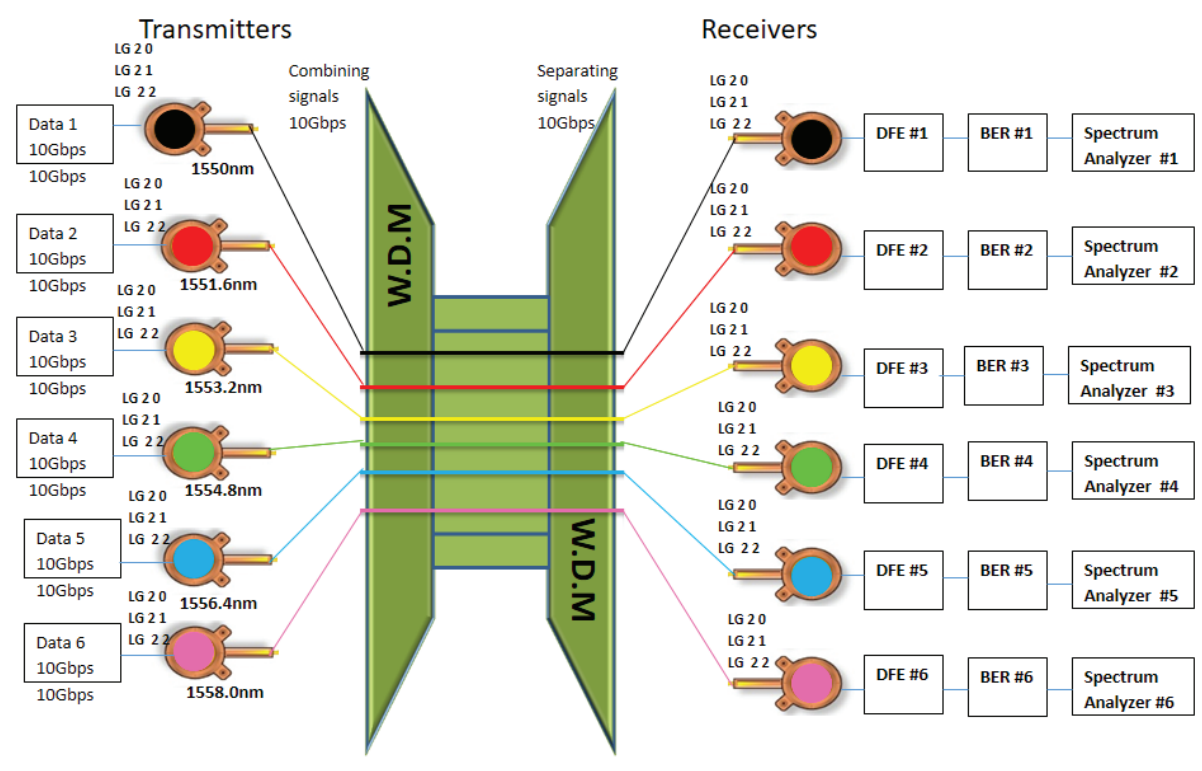

Fig. 1. Schematic diagram for Decision Feedback Equalization in DQPSK MWDM system using MMF.

space-time block coding exploiting space and delay diversity [27], constant modulus algorithm [28] and multi-modulus blind equalization [29]. RLS is preferable for next-generation MDM systems with faster convergence time compared to LMS.

\section{Contribution}

In contrast to the aforementioned equalization schemes, in this paper, electronic dispersion compensation (EDC) using decision feedback equalization (DFE) is numerically investigated for mode-wavelength division multiplexing (MWDM) in MMF. The objective of this paper is to analyze the relationship between different configuration of taps in the DFE and the resulting BER in order to establish the ideal combination of taps to restore the original signal after distortion due to mode coupling in a 60Gbps DQPSK WDM-MDM system through a $2.5 \mathrm{~km}$ MMF. In DFE, fixed tap length is conventionally used in noise control. To cancel the undesired noise from a primary noise source, the equalizer compensates for the uncertain effects after the receiver. Moving the DFE to the receiver side has several advantages. First, the Transmitter design is simplified, enabling a low-power design. Second, the equalizer coefficients can directly be adjusted at the receiver end, where they can be measured, which does not require a back-channel to the transmitter.

\section{MWDM System Design with DFE in MMF}

Fig. 1 shows the proposed design using DFE for a MWDM system propagating independent data on three modes per wavelength for a total of six wavelengths modulated using differential quadrature phase-shift keying (DQPSK). The proposed equalizer for the system is modeled using Synopsis OptSim optical communications suite [30]. Each channel is connected to a DFE which parameters include feed forward taps number and feedback taps number. Various combination of taps produce a corresponding bit error rate (BER) and this study shall establish the four best tap combinations that will produce satisfactory BER in the $2.5 \mathrm{~km} 60 \mathrm{Gbps}$ DQPSK MWDM optical system.

The transmitter consists of six spatial vertical cavity surface-emitting laser (VCSEL) arrays which are incorporated in the DQPSK transmitter and are directly modulated by an electrical signal. The VCSEL is driven by pseudo-random binary sequence (PRBS) electrical signals and modulated to non-return-to-zero pulses at its input and a fixed bias current. The six VCSELS are centered on wavelengths $1550 \mathrm{~nm}, 1551.6 \mathrm{~nm}, 1553.2$ $\mathrm{nm}, 1554.8 \mathrm{~nm}, 1556.4$ and $1558.0 \mathrm{~nm}$. Each VCSELs generate a laser beam composed of three different Laguerre-Gaussian modes LG 2 0, LG 21 and LG 22. The output of all VCSELs are multiplexed and propagated through a parabolic graded MMF with a radius of $25 \mu \mathrm{m}$ spanning $2.5 \mathrm{~km}$ in length. 
Table 1. DFE feed forward and feedback tap combinations under investigation

\begin{tabular}{|c|c|}
\hline $\begin{array}{c}\text { Feed Forward } \\
\text { Taps Number }\end{array}$ & $\begin{array}{c}\text { Feedback Tap } \\
\text { Number }\end{array}$ \\
\hline 10 & 5 \\
\hline 14 & 0 \\
\hline 15 & 6 \\
\hline 15 & 10 \\
\hline 1 & 0 \\
\hline 2 & 2 \\
\hline 5 & 2 \\
\hline 11 & 11 \\
\hline
\end{tabular}

Coupling between modes is modelled by a coupling matrix [31] :

$$
C=\left[\begin{array}{cccc}
c_{i, k} & c_{i, k+1} & \ldots & c_{i, n} \\
c_{i+1, k} & c_{i+1, k+1} & \ldots & c_{i+1, n} \\
\ldots & \ldots & \ldots & \ldots . \\
c_{m, k} & c_{m, k+1} & \ldots & c_{m, n}
\end{array}\right]
$$

where $i$ describes the source mode, $k$ describes the destination mode, $m$ describes the highest source mode and $n$ describes the highest destination mode. At the receiver side, a demultiplexer splits the power from the signals to six DQPSK spatial photodetectors which are each connected to a DFE. The DFE structure is based on transversal finiteimpulse response (FIR) filter. The signal transformation is described by the following equation:

$$
y(t)=\sum_{d=1}^{N} C_{d} x(t-i \Delta T)
$$

where $x(t)$ is incoming signal, $y(t)$ is the output signal, $d$ is the tap number, $N$ is the total number of taps, $C_{d}$ is the weight coefficients for the $d$-th tap and $\Delta T$ is the tap delay.

\section{Simulation Results}

The DFE corrects the received modal distribution in association with a priori modal distribution. The feedforward and feedback tap combinations under investigation are shown in Table 1. The output from each DFE is analyzed to determine the improvement in the BER.

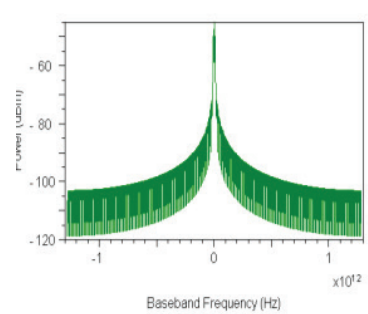

(a)

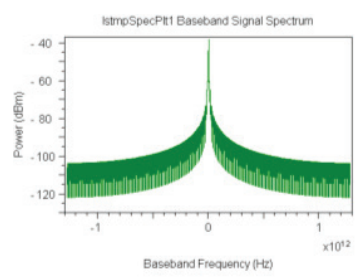

(c)

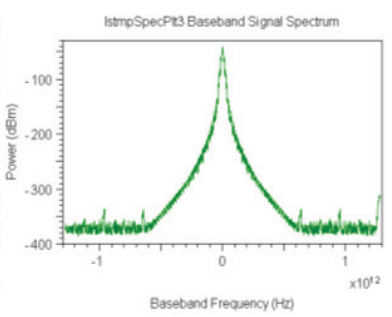

(b)

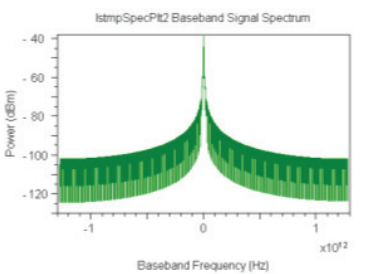

(d)
Fig. 2. Spectrum Analyzer diagrams for various tap combinations: (a) $(10,5),(b)(14,0),(c)(15,6)$ and (d) $(15,10)$

The analysis on several combinations of feed forward tap number and feedback tap number in the decision feedback equalizer revealed that when the set of $(10,5),(14,0),(15,6)$ and $(15,10)$ feed forward-feedback taps were employed, satisfactory BER was achieved as compared to many other combinations and in effect, significantly mitigated mode coupling. This led to the best DMG confinement and lowest BER. The spectrum analyzer diagrams for these combinations are shown in Fig. 2. The lowest noise levels were found to be $120 \mathrm{dBm}$ and the highest peaked at $-40 \mathrm{dBm}$ making the system highly efficient in terms of signal loss. Comparing the spectrum analyser diagrams in Fig.2 it is evident that the decision feedback equalizer with the set of $(10,5)$ feed forward-feedback taps achieved the least signal power loss with $45.342 \mathrm{dBm}$ compared to the corresponding other 3 each exhibiting signal power losses of $-38.63 \mathrm{dBm}$, $40.137 \mathrm{dBm}$ and $-37.8 \mathrm{dBm}$. The smaller the signal power loss the better the performance of the mode transmission system.

The BER based on the feed forward and feedback tap numbers are shown in Table 2.60Gbps data transmission was successfully achieved at a BER of $1.1519 \times 10^{-29}$ for feed forward - feedback tap combination $(10,5), 1.2769 \times 10^{-26}$ for feed forward - feedback tap combination $(14,0), 2.33 \times 10^{-23}$ for feed forward - feedback tap combination $(15,6)$ and $1.16 \times 10^{-35}$ for feed forward - feedback tap combination $(15,10)$ for a six-channel WDM-MDM system at a central wavelength of $1550 \mathrm{~nm}$ and $10 \mathrm{Gbps}$ per channel. However, feed forward feedback tap combinations $(1,0)(2,2)(5,2)$ and $(11,11)$ yielded the worst BERs ranging from 
$5.1974 \times 10^{-3}$ to $5.2624 \times 10^{-11}$ which in effect are not acceptable BERs.

The results demonstrate satisfactory equalization of power in MMF in order to alleviate mode coupling and modal dispersion.

Table 2. Results on comparison of number of DFE taps and BER

\begin{tabular}{|c|c|c|}
\hline $\begin{array}{c}\text { Feed forward tap } \\
\text { number }\end{array}$ & $\begin{array}{c}\text { Feedback tap } \\
\text { number }\end{array}$ & BER \\
\hline 10 & 5 & $1.15 \times 10^{-29}$ \\
\hline 14 & 0 & $1.28 \times 10^{-26}$ \\
\hline 15 & 6 & $2.33 \times 10^{-23}$ \\
\hline 15 & 10 & $1.16 \times 10^{-35}$ \\
\hline
\end{tabular}

\section{Conclusion}

DFE for a DQPSK MWDM architecture using three modes LG 20 ,LG 21 and LG 22 and 6 wavelengths achieved the lowest BER at $2.33 \times 10^{-23}$ using 15 feed forward taps and 6 feedback taps. The DFE optical equalizer compensates for the effects of mode coupling and achieved 60Gbps through a $2.5 \mathrm{~km}$-long MMF a central wavelength of $1550 \mathrm{~nm}$. The DFE suppress higher order modes and effectively excites only four DMG per wavelength.

\section{References}

1. "Cisco White Papers," San Jose, California (2015)

2. Deloitte, Digital Democracy Survey (2016)

3. K. Nisar et al., Journal of Network and Computer Applications, 36, no. 2, pp. 933-948 (2013)

4. A. Amphawan et al., Network and Complex Systems, 2, no. 3,pp. $1-9$ (2012)

5. S. Chaudhary et al., Optik - Int. Journal for Light and Electron Optics, 125, no. 18, pp. 5196-5198 (2014)

6. H. Al-Khafaji et al., IEICE Electronics Express, 10, no. 5, p. 20130044 (2013)

7. S. Aneesh et al., Optical and Quantum Electronics, 48, no. 5, pp. 1-10 (2016)

8. A. Amphawan, Opt. Eng., 50, p. 102001 (2011)

9. N. Bozinovic et al., Science, 340, no. 6140, pp. 15451548 (2013)

10. J. Carpenter et al., J. Lightw. Technol., 30, no. 12, pp. $1978-1984(2012)$

11. A. Amphawan, Opt. Express, 19, no. 10, pp. 9056-9065 (2011)

12. A. Amphawan, J. Mod. Opt., 59, no. 5, pp. 460-469 (2012)

13. Y. Wu et al., Opt. Express, 23, no. 16, pp. 20867-20875 (2015)
14. T. Sakamoto et al., J. Lightw. Technol., 33, no. 6, pp. 1175-1181 (2015)

15. B. J. Puttnam et al., 201621 st European Conference on Networks and Optical Communications (NOC), pp. 2934 (2016)

16. K. J. Park et al., Opt. Express, 24, no. 4, pp. 3543-3549 (2016)

17. D. Lee et al., J. Lightw. Technol., 34, no. 8, pp. 17541761 (2016)

18. A. Amphawan et al., J. Mod. Opt., 60, no. 20, pp. 16751683 (2013)

19. A. Amphawan et al., Int. Conf. on Opt. and Photon. Engineering (icOPEN), 9524: SPIE (2015)

20. Y. Fazea et al., J. Optical Commun. vol. 36, pp. 327333 (2015)

21. A. Amphawan et al., Journal of the European Optical Society-Rapid Publications, 12, no. 1, p. 12 (2016)

22. S. Ö. Arık et al., J. Lightw. Technol., 34, no. 11, pp. 2867-2880 (2016)

23. A. Amphawan et al., J. Mod. Opt., 50, no. 20, pp. 1745$1752(2012)$

24. P. J. Winzer, 39th European Conference and Exhibition on Optical Communication (ECOC 2013), pp. 1-4 (2013)

25. K. Choutagunta et al., Journal of Lightwave Technology, 35, no. 12, pp. 2451-2463 (2017)

26. Y. Weng et al., Optical Fiber Technology, 36, pp. 155180 (2017)

27. Y. Weng et al., Optical Fiber Technology, 36, pp. 231$236(2017)$

28. X. Li et al., Optical Fiber Communication Conference, Los Angeles, California, p. W2A.45: Optical Society of America (2017)

29. Y. Ren et al., IEEE Transactions on Wireless Communications, 16, no. 5, pp. 3151-3161 (2017)

30. RSoft, "OptSim 5.2," ed (2010)

31. G. Li et al., Advances in Optics and Photonics, 6, no. 4, pp. 413-487 (2014) 